\title{
Effects of Lorazepam and Citalopram on Human Defensive Reactions: Ethopharmacological Differentiation of Fear and Anxiety
}

\author{
Adam M. Perkins, ${ }^{1}$ Ulrich Ettinger, ${ }^{2,3}$ Robert Davis, ${ }^{4}$ Russell Foster, ${ }^{5}$ Steven C. R. Williams, ${ }^{1}$ and Philip J. Corr ${ }^{6}$ \\ ${ }^{1}$ Centre for Neuroimaging Sciences, Institute of Psychiatry, London SE5 8AF, United Kingdom, Departments of ${ }^{2}$ Psychiatry and ${ }^{3}$ Psychology, Ludwig \\ Maximilians University, 80539 Munich, Germany, ${ }^{4}$ Department of Psychology, Goldsmiths College, University of London, New Cross SE14 6NW, United \\ Kingdom, ${ }^{5}$ Institute of Liver Studies, King's College Hospital, London SE5 9PJ, United Kingdom, and ${ }^{6}$ School of Social Work and Psychology, University of \\ East Anglia, Norwich NR4 7TJ, United Kingdom
}

Drugs that are clinically effective against generalized anxiety disorder preferentially alter rodent risk assessment behavior, whereas drugs that are clinically effective against panic disorder preferentially alter rodent flight behavior. The theoretical principle of "defensive direction" explains the pattern of associations between emotion and defensive behavior in terms of the differing functional demands arising from cautious approach to threat (anxiety) versus departure from threat (fear), offering the prospect that clinically important emotions may be explained using a single rubric of defense. We used a within-subjects, placebo-controlled, design to test this theory, measuring the effects of citalopram and lorazepam on the defensive behavior of 30 healthy adult male humans. We indexed human defensive behavior with a translation of an active avoidance task used to measure rodent defense and found that lorazepam significantly reduced the intensity of defensive behavior during approach to threat (hypothetically anxiety-related) but not departure from threat (hypothetically fear-related). Contrary to prediction, citalopram did not affect either form of defensive reaction. Since lorazepam is a drug with well established anxiety reducing properties, these data support the hypothesis that anxiety is an emotion elicited by threat stimuli that require approach. These data also contribute to the validation of a novel human analog of an established experimental model of rodent fear and anxiety.

\section{Introduction}

Rodent studies suggest that risk assessment and flight are key components of the innate defensive repertoire of mammals (Blanchard, 1997). Risk assessment behavior is typically elicited by ambiguous threats such as suspicious noises or odors (Blanchard et al., 2003) whereas flight behavior is typically elicited by clear threat (e.g., a predator) in situations where an escape route is available (Blanchard et al., 1989). Ethopharmacological analyses of rodent defense (i.e., studies of drug effects on defensive behavior in naturalistic but controlled settings) associate these two behavior patterns with the emotions of anxiety and fear, respectively (Blanchard and Blanchard, 2008), on the basis that anxiolytic drugs (which are successful in the treatment of human anxiety) alter risk assessment (D. C. Blanchard et al., 1990), whereas panicolytic drugs (which are successful in the treatment of human fear/panic) alter flight (Griebel et al., 1995a).

The alteration of rodent defensive behavior by drugs that alter human emotional states not only points to a defense-based ex-

Received June 9, 2009; revised Aug. 4, 2009; accepted Aug. 27, 2009.

This work was supported by a British Academy grant (LRG-45458) to P.J.C. U.E. is supported by the Deutsche Forschungsgemeinschaft (ET 31/2-1). We thank S. Nelson for assisting with participant management and G. Ivin for providing pharmacy support.

Correspondence should be addressed to Adam Perkins, Centre for Neuroimaging Sciences, Institute of Psychiatry, Box P089, De Crespigny Park, London SE5 8AF, UK. E-mail: Adam.Perkins@kcl.ac.uk.

DOI:10.1523/JNEUROSCI.2696-09.2009

Copyright $\odot 2009$ Society for Neuroscience ～0270-6474/09/2912617-08\$15.00/0 planation for some affective disorders (Blanchard et al., 2001), but also has allowed these rodent defense findings to be integrated theoretically with the findings of earlier studies that examined the effects of such drugs on rodent behavior in classical learning tasks. More specifically, the capacity of anxiolytic drugs systematically to alter rodent behavior in goal conflict tasks that are seemingly threat-free [e.g., delayed matching (Tan et al., 1990)] has been cited as evidence that anxiety is a response to goal conflict rather than ambiguous threat per se (Gray and McNaughton, 2000). According to this theory, a rodent's need to investigate an ambiguous threat to verify its dangerousness putatively generates an approach-avoidance goal conflict that, in turn, elicits risk assessment accompanied by anxiety (McNaughton and Corr, 2004). Emerging from this work is a theoretical principle known as "defensive direction," which postulates that anxiety is elicited by threats requiring approach (i.e., that cause goal conflict) and fear by threats that need not be approached, but simply avoided (McNaughton and Corr, 2004; Corr, 2008).

We tested this theory by examining drug effects on human behavior in a computerized translation of a rodent runway task that has been used for ethopharmacological studies, namely the mouse defense test battery (MDTB) (Griebel et al., 1997) (see Fig. $1 A)$. The MDTB was selected as the basis for the present research as it can be configured so that the mouse is trapped in a closed runway or free to move along an endless runway. This dual configuration design permits the systematic measurement of drug 
effects on behavior during approach to threat (i.e., the putative eliciting stimulus for anxiety/risk assessment) and departure from threat (i.e., the putative eliciting stimulus for fear/flight), rendering it suitable for a pharmacological test of the principle of defensive direction.

In our human translation of the MDTB we measured the effects of citalopram, lorazepam, and placebo on human behavior in both MDTB configurations (Fig. $1 B, C$ ) to test the idea that anxiety is elicited by threats requiring approach and fear by threats that need not be approached. We accordingly hypothesized that lorazepam, a benzodiazepine drug with well established anxiolytic properties (e.g., Gould et al., 1997), would alter behavior during approach to threat but not departure from threat. Conversely we hypothesized that citalopram, a selective serotonin reuptake inhibitor (SSRI) with well established panicolytic properties (Wade et al., 1997), would alter behavior during departure from threat but not approach to threat.

\section{Materials and Methods}

\section{Participants}

All participants gave written informed consent before being enrolled. The study was approved by the research ethics committee of King's College Hospital in London, UK. Thirty healthy male participants (mean age $=25.8, \mathrm{SD}=6.0$, age range $18-40$ years) completed the study via a two-stage screening process: all initial respondents to advertisements for the study were screened by telephone using a detailed medical checklist, with those that passed undergoing a face-to-face semistructured clinical interview with a consultant psychiatrist (lasting $\sim 40-60$ $\mathrm{min})$. This interview explored the participant's medical history in depth to determine their suitability for the study. Exclusion criteria included psychiatric illness, serious head injury, dyslexia, learning difficulties, drug addiction/alcoholism, current smoking, current heavy coffee drinking, recent surgery, major medical disorders, or taking prescribed or over-the-counter medication of any kind. Participants were also required to have a body mass index (BMI) between 19 and 25, be aged between 18 and 40 years old, and to be native English speakers. These latter inclusion criteria were applied to ensure that all participants had approximately equal physical capability to operate the behavioral task and could understand adequately the task instructions and questionnaires. We restricted our sample to male participants to avoid potentially confounding effects of menstruation-related hormonal fluctuations during the month long testing window (Kumari and Corr, 1998).

Before each pharmacological testing session each participant underwent an additional brief medical review in which he was asked by the consultant psychiatrist about any changes in his health since screening, including new use of medication, alcohol, caffeine, or tobacco in the previous $48 \mathrm{~h}$. If the physician judged that it was appropriate to proceed with dosing, baseline vital signs (pulse and blood pressure) were recorded and the participant ingested the drug. Following completion of each pharmacological testing session, each participant was asked whether or not he thought he had received an active drug or the placebo and then underwent a posttesting medical review including vital signs measurement to verify that he was fit for discharge.

\section{Personality questionnaires}

In rodents, the direction of anxiolytic drug effects upon risk assessment behavior is determined by the animals' state level of defensiveness: in mildly threatened rodents (e.g., that have been exposed to the odor of a predator) risk assessment behavior is typically reduced by anxiolytic drugs (R. J. Blanchard et al., 1990), whereas in severely threatened rodents (e.g., that are in clear sight of a nearby predator) anxiolytic drugs typically increase risk assessment (D. C. Blanchard et al., 1990). This bidirectional pattern of pharmacological effects has been interpreted as reflecting the capacity of anxiolytic drugs to reduce the perceived intensity of a threat (Blanchard et al., 2003). Thus, in a mildly threatened rodent, an anxiolytic-induced reduction in perceived threat intensity is signaled by a decrease in risk assessment behavior. In severely threatened rodents, where more intensively defensive behavior patterns such as freezing are prevalent, an anxiolytic-induced reduction in perceived threat intensity is indicated by the onset of risk assessment (Blanchard et al., 2003).

In our human translation of the MDTB, unlike the rodent version, the participants were at no stage exposed to a deadly threat such as a predator and additionally were made aware before participation that they were in a tightly controlled experiment during which they could not be injured in any way. At face value, therefore, the average state level of perceived threat among our participants was more comparable to that of mildly threatened rodents rather than that of severely threatened rodent subjects. If correct, this analysis suggests that anxiolytic drugs should not exert a bidirectional effect upon risk assessment behavior, but instead 


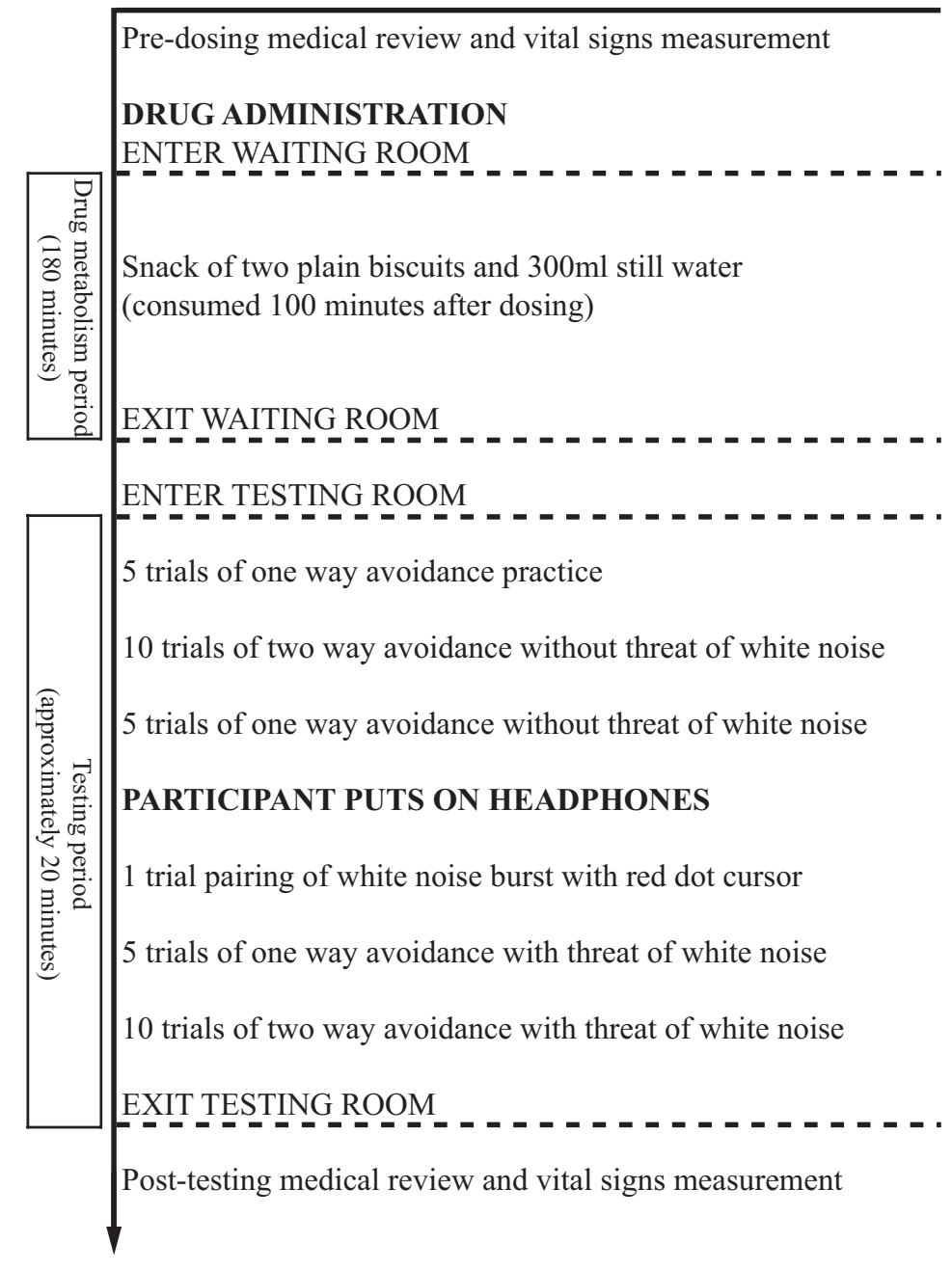

Figure 2. Program for pharmacological testing session.

will reduce risk assessment, as is seen in mildly threatened rodents (R. J. Blanchard et al., 1990). However, human trait individual differences in perceptions of threat intensity have been likened to state individual differences in rodent perceived threat (McNaughton and Corr, 2004), meaning that the effect of anxiolytic drugs upon risk assessment behavior may be modulated to some extent by individual differences in perceived threat intensity.

To conduct a preliminary test of this possibility, we measured trait individual differences in perceived intensity of threat in our participants using questionnaire self report. Since a validated questionnaire measure of this construct has not yet been created, we administered the Fear Survey Schedule (FSS) (Wolpe and Lang, 1977) on the basis that preliminary investigations of human defensive behavior using written threat scenarios suggest that the FSS has some capacity to measure perceived threat intensity (Perkins and Corr, 2006; Perkins et al., 2010).

The FSS contains two major subscales: social fear, which psychometric analyses suggest is a hybrid measure of trait anxiety and fear (Cooper et al., 2007), and tissue damage fear, which psychometric evidence suggests is a relatively pure measure of fear (Perkins et al., 2007). These subscales have not yet been differentiated pharmacologically and so, as a precaution, we administered both of them to our participants as potential measures of perceived threat intensity. In addition, because trait individual differences in perceived intensity of threat have been likened for theoretical reasons to individual differences in trait anxiety (McNaughton and Corr, 2004), we also administered a well established questionnaire measure of this construct, namely the Y2 (trait) scale from the Spielberger State-Trait Anxiety Inventory (STAI) (Spielberger et al., 1983).
To assess whether or not outcomes could be altered by individual differences in how aversive the participants found the task, a brief affect questionnaire [the short scale Positive Affect and Negative Affect Schedule (Mackinnon et al., 1999)] was administered immediately after the first completion of the behavioral task. This questionnaire contains five negative and five positive affect-related words (e.g., "distressed," "enthusiastic," "upset") that the participants rated on a five point scale to show how they were feeling at that time. Scores on the five negative items were totaled to provide an indication of how aversive each participant found the task.

\section{Experimental paradigm}

Methods. This study used a placebo-controlled, double blind, within-subjects, repeated measures design in which pharmacological effects on human defensive behavior were measured in three different counterbalanced drug conditions [panicolytic (10 mg of citalopram), anxiolytic (1 mg of lorazepam), and placebo (50 mg of ascorbic acid)] in three separate testing sessions, $7 \mathrm{~d}$ apart to ensure drug washout. The drug doses were selected on the basis of previous research showing that they produce a significant affective response with minimal side effects (Kuepper et al., 2006). The order in which participants received the drugs was set by a six group randomization schedule. Each pharmacological testing session had the same structure (Fig. 2).

Behavioral task. We measured human defensive behavior by translating the MDTB into the form of a joystick-operated runway task (JORT). In the MDTB, rodents are video recorded as they respond to different types of threat so that their behavior may be scored afterward. Our human version of the MDTB scored behavior automatically by computer allowing the experiment to be run without need for video analysis.

Apparatus. Behavioral data were acquired using a force-sensing joystick apparatus (PH-JS1, Psyal, London, UK). The participant sat on a stool that was integral to the apparatus and operated the joystick handle while facing a white screen measuring approx. $1.5 \times 1.5 \mathrm{~m}$. A vertically orientated image of a runway was projected on this screen (Fig. $1 B, C$ ) by a purpose-written computer program (PS-JS1, Psyal, London, UK).

Testing procedure. The participant pushed the joystick handle forward with both hands to move a cursor (a circular green dot) along the onscreen runway ahead of a second, computer controlled pursuing cursor (a circular red dot). In order for it to remain visible at all times, the green dot cursor representing the participant remained motionless in the center of the runway, with a visual impression of speed along the runway being created by scrolling the background downward at a speed directly proportional to the force applied to the joystick handle. A reduction in force applied to the joystick handle slowed the green dot cursor and allowed the red dot cursor to catch up, but the participant could not move it backwards by pulling on the handle. The only other person in the room during the testing session was the experimenter (A.P.) who was seated behind the participant out of their line of sight. Computer controlled instructions for each phase of the task were displayed on screen: the experimenter did not speak during testing, unless the participant required the experimental procedure to be clarified.

Seven days before the start of the pharmacological testing sessions, the maximum physical strength of each participant was recorded in a rehearsal testing session. In this session, before attempting the avoidance task, the participant was required to push the joystick handle five times 
with both hands as hard as possible while seated on the joystick stool. The minimum force required for the green dot cursor to reach escape velocity on the runway for all subsequent pharmacological testing sessions involving a participant was set at $50 \%$ of whatever maximum force that he exerted during calibration. This procedure meant that in principle each participant had to work equally hard to outrun the pursuing red dot cursor relative to other participants, even if they differed in absolute physical strength. This procedure also meant that each participant had to work equally hard across the three pharmacological testing sessions. By coupling runway velocity to physical effort we increased the naturalism of our task by ensuring that avoidance of threat by our participants had significant calorie cost, mimicking that incurred by animals in real threat situations. The calibration level of 50\% maximum strength was determined during extensive piloting to ensure that the participant must exert non-trivial physical effort in return for avoidance of the pursuing stimuli, without suffering severe fatigue that might confound results over consecutive trials.

To accommodate a wide range of individual differences in physical strength, the joystick mechanism was designed to measure from $0-500 \mathrm{~N}$ of force applied to the handle. The handle was made out of a solid steel rod $25 \mathrm{~mm}$ in diameter to resist bending or breaking during use. By mounting the joystick on a stool, the participant's weight served to pin the apparatus firmly to the floor even during violent avoidance maneuvers. A soft rubber hand grip was fitted to the joystick handle.

Prethreat. Each pharmacological testing session began with 5 practice trials of one-way active avoidance (Fig. $1 C$ ) that allowed the participant to acquaint himself with the coupling of the movement of the joystick handle with onscreen movement of the green dot cursor. The participant then completed 10 trials of two-way active avoidance. The format for the two-way active avoidance trials was identical to that of the one-way active avoidance trials except for the presence of a second red dot cursor traveling along the runway ahead of the green dot cursor (Fig. $1 B$ ). Since movement of the green dot cursor away from one red dot cursor automatically moved it closer to the other (both red dot cursors moved in the same direction), the participant was thus trapped between the two red dot cursors in a two-way active avoidance goal conflict situation for the duration of the trial, regardless of whether or not he slowed or accelerated along the runway. The participant then completed 5 more trials of oneway active avoidance.

Threat. The participant put on a pair of headphones and watched an example trial on the screen where the green dot cursor was pursued and then contacted by the red dot cursor upon which a $250 \mathrm{~ms}, 115 \mathrm{~dB}$ burst of white noise was presented (the joystick was disabled for this phase so that all participants received the same exposure to the white noise). The participant then completed 5 test trials of one-way active avoidance that were identical to the prethreat trials except that the participant received a $115 \mathrm{~dB}$ white noise burst whenever he failed to move the green dot cursor fast enough to avoid the pursuing red dot. Finally participants completed a further 10 trials of two-way active avoidance, also under threat of white noise (contact between the green dot and either of the red dots triggered the white noise burst). To prevent prolonged exposure to white noise, a trial terminated automatically as soon as the participant had received a single $250 \mathrm{~ms}, 115 \mathrm{~dB}$ burst of white noise. If the participant successfully avoided the threat stimuli for $7 \mathrm{~s}$ the trial automatically terminated. To enhance unpredictability, intertrial intervals were varied pseudorandomly between 15 and $30 \mathrm{~s}$. The software controlling the joystickoperated runway task automatically recorded the velocity (pixels/s) of the green dot cursor every $10 \mathrm{~ms}$ throughout the testing session. During intertrial intervals when no force was applied to the joystick handle the velocity was zero.

Behavioral data analysis. Approach-withdrawal oscillation in the closed runway configuration of the MDTB is described as part of rodent risk assessment behavior (Griebel et al., 1995b). A link between anxiety and approach-withdrawal oscillation in the MDTB is suggested by the sensitivity of this behavior to anxiolytic drugs (Griebel et al., 1998; Stemmelin et al., 2008). In line with this literature, we used the magnitude of approach-withdrawal oscillations (measured as the SD of the average velocity of the participants' cursor during two-way active avoidance) as our putative measure of risk assessment intensity in the computerized runway task. In the MDTB escape speed is regarded as a measure of rodent flight behavior (Griebel et al., 1995a): in the present study we conceptualized the average velocity of the participants' cursor during one-way active avoidance in the endless runway configuration as our putative measure of the intensity of flight behavior. To control for drug effects on motor function, the behavior of each participant during oneway and two-way active avoidance was measured before $(x)$ and after $(y)$ introduction of a threat stimulus: the pure effect of threat on behavior in both parts of the runway task could therefore be calculated as $y-x$ for each of the dependent variables.

The dependent variable that we labeled as risk assessment intensity was, therefore, measured as the degree to which the introduction of the threat of a white noise burst altered the magnitude of approachwithdrawal oscillation during two-way active avoidance (e.g., average oscillation in the 10 two-way active avoidance trials before exposure to threat stimulus subtracted from average oscillation in 10 conflict trials after exposure to threat stimulus). For the same reason, the dependent variable that we labeled as flight intensity was measured behaviorally as the degree to which the introduction of the threat of a white noise burst altered the velocity of the green dot cursor along the runway during one-way active avoidance (i.e., average velocity in the one-way active avoidance trial immediately before exposure to threat stimulus subtracted from average velocity in the one-way active avoidance trial immediately after exposure to threat stimulus).

Statistical analyses. Statistical analyses were performed using the Statistical Package for the Social Sciences (SPSS). Associations between personality variables and dependent variables were assessed using Pearson's product-moment correlation coefficient to determine covariate eligibility. Effects of drugs and personality upon task performance were analyzed by repeated measures ANCOVA, in which Drug (citalopram, lorazepam, placebo) formed a three level within-subjects factor. Any personality score that met the established covariance criteria [i.e., questionnaire scores that correlated significantly with a dependent variable (Tabachnick and Fidell, 2007)] was entered as a covariate. Simple contrasts were used to test for specific drug effects against placebo. The Pearson $\chi^{2}$ test was used to determine whether or not participants could detect ingestion of an active drug by self report. The level for statistical significance was set to $p=0.05$.

\section{Results}

Table 1 presents descriptive statistics and correlations among questionnaire measures of personality and behavioral criteria. The pattern of correlations between trait anxiety and fear questionnaire scores parallels previous psychometric findings (Cooper et al., 2007): FSS social fear more closely resembles trait anxiety than does FSS tissue damage fear. No statistically significant correlations emerged between ratings of task aversiveness and psychometric variables; however, task aversiveness was significantly positively correlated with flight intensity in the placebo condition. This finding provides circumstantial support for the face validity of the JORT by suggesting that participants found the white noise burst threat stimulus to be aversive and that this aversiveness was sufficiently potent to influence their flight behavior in the expected direction. There were no statistically significant correlations among the behavioral criteria, but questionnaire scores on FSS social fear were significantly positively correlated with risk assessment intensity in the Lorazepam condition only, indicating that it is eligible for inclusion as a covariate of interest in analyses of drug effects on risk assessment intensity.

\section{Drug effects on task performance}

Analysis of covariance with repeated measures and FSS social fear as covariate showed a significant main effect of Drug on risk assessment intensity $\left(F_{(2,56)}=3.82 ; p=0.028\right)$ (Fig. $3 A$ ). Simple contrasts showed that this drug effect on risk assessment was 
Table 1. Descriptive statistics and inter-correlations for questionnaire measures of personality, negative affect, and behavioral criteria

\begin{tabular}{|c|c|c|c|c|c|c|c|c|c|c|c|}
\hline Variable & Mean (SD) & 1 & 2 & 3 & 4 & 5 & 6 & 7 & 8 & 9 & 10 \\
\hline 1. Trait anxiety & $42.23(8.98)$ & - & & & & & & & & & \\
\hline 2. Tissue damage fear & $28.07(13.04)$ & 0.267 & - & & & & & & & & \\
\hline 3. Social fear & $38.77(17.92)$ & $0.383^{*}$ & $0.597^{* *}$ & - & & & & & & & \\
\hline 4. Task aversiveness & $6.30(1.73)$ & -0.018 & 0.134 & 0.034 & - & & & & & & \\
\hline 5. Flight intensity (Citalopram) & $-0.30(0.10)$ & -0.168 & -0.020 & -0.303 & -0.059 & - & & & & & \\
\hline 6. Flight intensity (Lorazepam) & $-0.18(0.97)$ & -0.127 & -0.133 & -0.015 & 0.192 & 0.092 & - & & & & \\
\hline 7. Flight intensity (Placebo) & $-0.06(0.56)$ & 0.100 & 0.077 & 0.211 & $0.365^{*}$ & -0.112 & 0.335 & - & & & \\
\hline 8. Risk assessment intensity (Citalopram & $-0.01(0.06)$ & -0.110 & 0.188 & 0.109 & 0.197 & 0.145 & 0.305 & -0.005 & - & & \\
\hline 9. Risk assessment intensity (Lorazepam) & $-0.03(0.08)$ & -0.051 & 0.240 & $0.483^{* *}$ & -0.001 & -0.001 & 0.165 & 0.092 & 0.324 & - & \\
\hline 10. Risk assessment intensity (Placebo) & $-0.01(0.06)$ & 0.187 & -0.123 & 0.081 & -0.244 & -0.218 & 0.072 & -0.184 & 0.140 & -0.037 & - \\
\hline
\end{tabular}

$N=30 .{ }^{*} p<0.05 ; * * 0.01$.
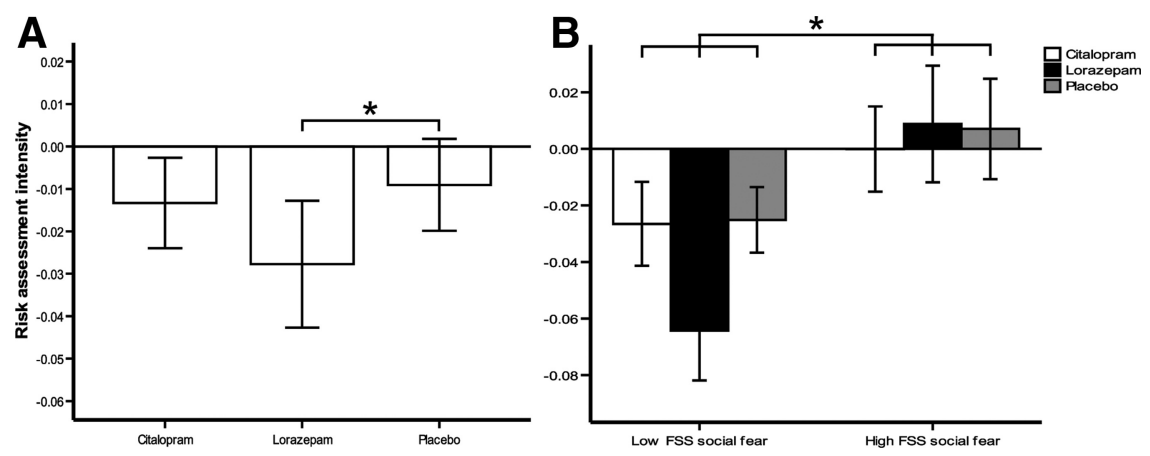

Figure 3. Behavioral data showing effects of drugs and personality on risk assessment intensity (error bars represent 1 $\mathrm{SEM})$. $\boldsymbol{A}$ shows that risk assessment intensity was significantly reduced by lorazepam but that citalopram was not significantly different from placebo in its effect on risk assessment intensity. $\boldsymbol{B}$ shows that risk assessment intensity was significantly increased by high levels of FSS social fear across all three drug conditions. In addition, $\boldsymbol{B}$ suggests that the effect of lorazepam on risk assessment intensity was driven by participants scoring in the lower half of the sample on FSS social fear (interaction: $p=0.055$ ) and participants scoring in the upper half of the sample on FSS social fear displayed a significantly high level of resistance to the effects of lorazepam. ${ }^{*} p<0.05$.

caused by lorazepam, as it significantly reduced risk assessment intensity relative to placebo $\left(F_{(1,28)}=4.68, p=0.039\right)$ whereas citalopram did $\operatorname{not}\left(F_{(1,28)}=0.49 ; p=.826\right)$. There was no statistically significant main effect of drug condition on flight intensity $\left(F_{(2,56)}=\right.$ $0.51 ; p=0.601)$. There was no significant interaction between drug condition and ratings of how aversive participants found the $\operatorname{task}\left(F_{(2,56)}=0.91 ; p=0.407\right)$.

\section{Personality effects on task performance}

As a preliminary contribution toward the ongoing discussion of comparability between state emotions in animals and affective traits in humans (McNaughton and Corr, 2004; Corr, 2008), we also explored personality effects on task performance and found that FSS social fear exerted a statistically significant betweensubjects main effect on risk assessment intensity $\left(F_{(1,28)}=4.88\right.$; $p=0.036)$. Participants scoring in the upper half of the sample on FSS social fear displayed higher risk assessment intensity in all drug conditions than participants scoring in the lower half of the sample on FSS social fear (Fig. 3B). Among participants scoring in the lower half of the sample on social fear, lorazepam appeared to exert an especially strong reducing effect on risk assessment intensity (Fig. $3 B$ ), although the FSS-by-Drug interaction narrowly failed to reach statistical significance $\left(F_{(2,56)}=3.06 ; p=\right.$ $0.055)$. There was no statistically significant main effect of FSS social fear on flight intensity $\left(F_{(1,28)}=0.42 ; p=0.523\right)$; and nor was there a significant interaction FSS-by-drug interaction $\left(F_{(2,56)}=2.04 ; p=0.140\right)$.
Drug effects on nonavoidance variables After each drug testing session the pulse and blood pressure of all participants were recorded. Drug condition exerted a significant main effect on pulse $\left(F_{(2,56)}=5.27\right.$; $p=0.008$ ), with pulse being elevated in the lorazepam condition relative to placebo $\left(F_{(1,28)}=7.18 ; p=0.012\right)$ but not in the citalopram condition $\left(F_{(1,28)}=0.131\right.$; $p=0.720)$. Drug condition had no significant effect on blood pressure $\left(F_{(2,56)}=\right.$ $0.87 ; p=0.426)$. After each drug testing session participants were asked their opinion as to whether or not they had received an active drug or a placebo. In the citalopram condition, 21 participants said they had received an active drug, 7 said they had received a placebo, and 2 said they did not know what they had received. Statistical testing showed that participants detected ingestion of an active drug at levels significantly above chance $\left(\chi^{2}(1)=19.40, p<0.001\right)$. In the lorazepam condition, 21 participants said they had received an active drug, 6 said they had received a placebo and 3 said they did not know what they had received. Statistical testing showed that participants successfully detected the ingestion of an active drug at levels significantly above chance $\left(\chi^{2}(1)=18.60, p<0.001\right)$. In the placebo condition, 9 participants said they had received an active drug, 15 said they had received a placebo and 6 said they did not know what they had received. Statistical testing showed that participants did not detect the placebo at levels significantly above chance $\left(\chi^{2}(1)=4.20, p=0.122\right)$.

\section{Discussion}

We show, first, that lorazepam significantly altered behavior during approach to threat but not during departure from threat. Since lorazepam is an anxiolytic drug (Gould et al., 1997), this finding contributes to the validation of the theoretical principle of defensive direction (McNaughton and Corr, 2004) that maintains anxiety is an emotion elicited by threats that require approach. The specific behavior that was altered by lorazepam (approach-withdrawal oscillation) is one of a class of defensive behaviors labeled as risk assessment that have been linked pharmacologically to anxiety in rodents (Griebel et al., 1998). By demonstrating an effect on approach-withdrawal oscillation of an anxiolytic compound in humans, our results are broadly consistent with earlier self-report questionnaire studies showing that the patterning of human defensive behavior parallels that of rodents (Blanchard et al., 2001; Perkins and Corr, 2006). 
More contentiously, our use of unambiguous threat stimuli could be interpreted as suggesting that the capacity of a threat stimulus to elicit anxiety does not depend upon it being ambiguous, as rodent defense findings might suggest (Blanchard et al., 2003), but instead upon whether or not it presents a goal conflict (Gray and McNaughton, 2000). This interpretation must, however, be viewed with caution because of the constrained nature of the defensive behaviors that were measured using the joystick-operated version of the MDTB: For example, rodent risk assessment behavior encompasses not only approachwithdrawal oscillation but also movements that facilitate environmental scanning, such as side-to-side head sweeps (Blanchard et al., 2003). We were unable to sample these latter phenomena by means of our joystick apparatus and so were unable to provide a test of drug effects upon the full repertoire of rodent-like risk assessment behaviors.

A further reason for caution when interpreting our lorazepamrelated results is provided by the finding that anxiolytic drugs have been found to increase approach-withdrawal oscillation in the MDTB (Griebel et al., 1998; Stemmelin et al., 2008), whereas we found that lorazepam reduced the intensity of approach-withdrawal oscillations in our participants. This opposite directional effect of anxiolytic drugs on approach-withdrawal oscillation in rodent and human data is difficult to explain with certainty. One possible answer stems from the discovery that anxiolytic drugs increase risk assessment in severely threatened rodents (D. C. Blanchard et al., 1990 ) but reduce it in mildly threatened subjects (R. J. Blanchard et al., 1990). Unlike MDTB subjects, our participants were not exposed to a deadly predator and so their average level of perceived threat should at face value have been more comparable to that of mildly threatened rodents than that of severely threatened rodent subjects. If correct, this analysis suggests that anxiolytic drugs should reduce risk assessment in our participants, as was observed.

As an additional caveat it must be noted that behavioral responses during the two-way avoidance phase have a dual effect, decreasing distance to one threat stimulus just as they increase distance to the other. Thus, although the threat stimuli used in our study were unambiguous, the responses they elicited could be construed as containing ambiguity. This interpretation suggests that considerable further research is required to clarify how the properties of a threat stimulus and the responses to it elicit anxiety.

The absence of a significant main effect of the panicolytic drug citalopram on approach-withdrawal oscillation could be cited as additional support for the notion that oscillation specifically relates to anxiety. However, contrary to expectations, citalopram also failed to exert a significant main effect on flight intensity during departure from threat. Viewed together, these two null outcomes for citalopram limit the firmness of conclusions that can be drawn concerning the relevance of this drug in the present research. One explanation for an absence of citalopram effects could be that, unlike benzodiazepine compounds, SSRI drugs such as citalopram typically require administration for several weeks before gaining clinical effectiveness (Wade et al., 1997). We were unable for ethical reasons to dose our participants chronically and so this may have rendered our single citalopram dose ineffective. Evidence on the acute effects of citalopram on rodent defense has not yet been obtained but ethopharmacological studies using other clinically effective panicolytic drugs such as imipramine and fluoxetine show that single doses of these compounds reliably alter flight behavior [although in the opposite direction to chronic dosing - potentiating rather than reducing flight; e.g., see the study by Griebel et al. (1995a)]. The use of a single citalo- pram dose in the present study was plausible as our aim was to show a selective effect of citalopram on flight behavior regardless of its direction. Results indicate that a single dose is ineffective.

The absence of citalopram effects on simple avoidance behavior could also be explained by the possibility that the defensive direction model (Gray and McNaughton, 2000; McNaughton and Corr, 2004) is incorrect with regard to fear. However, evidence from nondrug studies showing that fear prone individuals preferentially select threat responses entailing orientation away from threat (Perkins and Corr, 2006; Perkins et al., 2009) provides relatively clear support for the fear-related aspects of the theory and suggests that this explanation is not particularly credible.

An alternative explanation for the absence of citalopram effects on defensive behavior may be found in the low level of threat applied to participants in the present study: Whereas mice in the MDTB are exposed to what is to them a deadly threat (a rat) our participants were exposed to unpleasant but harmless white noise bursts. The rationale for such an explanation lies in the hypothesis that panic reactions and mild fear reactions are mediated by different brain regions (periaqueductal gray and ventral prefrontal stream respectively; McNaughton and Corr, 2004). If it is accepted that panic disorder is specifically characterized by recurrent episodes of intense fear (American Psychiatric Association, 1994), then citalopram, as an established panicolytic (Wade et al., 1997), may act more powerfully on the brain systems responsible for mediating intense fear than those that control mild fear. Thus the white noise threat stimulus in the present study may not have been sufficiently potent to prompt substantial activity in the brain systems responsible for causing the panic reactions that are modulated by SSRI's such as citalopram in both clinical settings and the MDTB.

This explanation points to a need for future research to use functional brain imaging techniques during human defense to distinguish the capacity of ethoexperimental tasks to activate relevant brain areas, perhaps using threat stimuli such as electric shocks that will most likely be perceived by participants as more potent than white noise. For example, Mobbs et al. (2007) found that as a virtual predator moved through a maze toward a cursor controlled by the participant, brain activity (as measured by functional magnetic resonance imaging) moved from the ventromedial prefrontal cortex to the periaqueductal gray. The predator delivered electric shocks to the participant if it caught the cursor, suggesting that this mode of punishment may be sufficiently aversive to cause a degree of panic.

Neuroimaging studies of this kind are especially relevant in tests of the defensive direction theory as it makes clear predictions concerning differential brain activity during simple avoidance and approach to threat. In particular the defensive direction theory maintains that the septo-hippocampal system is the seat of anxiety but not fear (Gray and McNaughton, 2000). Thus, if the JORT variables of flight intensity and oscillation during conflict are related to fear and anxiety respectively then the former should be distinguished from the latter by the addition of activity in the septo-hippocampal system to underlying amygdala activity. It is to be hoped that future research will test these predictions, preferably alongside tests of predictions from other important theories of defensive emotion (Deakin and Graeff, 1991).

A subsidiary aim of the present research was to investigate the notion that the effect of anxiolytic drugs upon human risk assessment behavior may be modulated by individual differences in perceived threat intensity. This possibility is suggested by the finding that the state level of defensiveness of a rodent alters the 
effect of anxiolytic drugs on its risk assessment behavior (R. J. Blanchard et al., 1990). Since human trait individual differences in perceptions of threat intensity have been likened to state individual differences in rodent defensiveness (McNaughton and Corr, 2004), we tested this notion using self-reports of trait differences in threat sensitivity. Results showed a trend level tendency for lorazepam to reduce risk assessment intensity more strongly in participants who scored in the lower half of the sample on FSS social fear. Since anxiolytic drugs reduce risk assessment in mildly threatened rodents but increase it in severely threatened rodents (Blanchard et al., 2003) these findings imply that analogies between the threat state of rodents and trait individual differences in perceived threat intensity in humans may be valid (McNaughton and Corr, 2004). For example, low scorers on FSS social fear may be likened to mildly threatened rodents and high scorers to animals that have perceived a relatively greater level of threat.

This idea is reinforced by the additional finding that participants scoring in the upper half of the sample on FSS social fear displayed significantly higher risk assessment intensity in all drug conditions than participants scoring in the lower half of the sample on FSS social fear. Since the two-way avoidance phase of the JORT was intended to cause a goal conflict, this latter finding is especially important as social situations at face value often contain goal conflict and the items in the FSS social fear scale reflect this (e.g., public speaking; entering a room where other people are already seated). The discovery that high scorers on this scale showed more intense physical reactions to goal conflict than low scorers therefore provides independent validation of the JORT as a measure of conflict sensitivity and hence anxiety.

These findings support the notion that neuroscientific results may be clarified by taking into account trait individual differences in personality (Kumari et al., 2004) and concur with previous psychometric findings that FSS scores may measure perceived intensity of threat (Perkins et al., 2009) but do not explain precisely why a questionnaire that purports to measure social fear should appear to modulate effects of an anxiolytic drug on defensive behavior in nonsocial situations. One explanation is provided by the recent finding that, for humans, fear acquired through social observation engages similar neural mechanisms to nonsocial classical fear conditioning (Olsson et al., 2007). This finding implies that the prototypical brain systems that control mammalian reactions to threat have, in humans, become substantially adapted for processing social hazards, and so individual differences in the functioning of such brain systems are likely to be expressed in humans most clearly as individual differences in susceptibility to social threat.

Despite this possibility, the necessity of using questionnaires not originally intended as measures of defensive reactions was a clear limitation for our study and so results support the notion that a new generation of psychometric measures may be required if defense-level personality traits are to be measured by means of questionnaire in future research of the present type (Corr, 2008). This need is illustrated especially clearly by the failure of scores on the Spielberger Trait Anxiety Inventory (Spielberger et al., 1983) to associate significantly with any defensive variable despite reasons to believe that they should (McNaughton and Corr, 2004).

Another limitation of our study stems from our use of male participants only. This was done to avoid confounding menstrual cycle effects. Since rodent females typically show greater defensiveness than males (Litvin et al., 2008) and human females are more prone than males to negative emotion (Farmer et al., 2003), had we been able to use female participants we might have obtained more powerful results.

In summary, we found evidence that anxiety may result from approach to threat as predicted by the principle of defensive direction. Participants' behavior in response to clear threat that must be approached was altered to a statistically significant degree by an anxiolytic drug. This drug did not significantly affect behavior during simple avoidance of threat. We also found a statistically significant main effect of personality upon threatapproach behavior that implies human defensive behavior is ultimately governed by a combination of situational and trait factors.

\section{References}

American Psychiatric Association (1994) Diagnostic and statistical manual of mental disorders (DSM-IV), Ed 4. Washington, DC: American Psychiatric Association.

Blanchard DC (1997) Stimulus and environmental control of defensive behaviors. In: The functional behaviorism of Robert C. Bolles: learning, motivation and cognition. (Bouton M, Fanselow M, eds), pp 283-305. Washington DC: American Psychological Association.

Blanchard DC, Blanchard RJ (2008) Defensive behaviors, fear and anxiety. In: Handbook of anxiety and fear (Blanchard RJ, Blanchard DC, Griebel G, Nutt D, eds), Vol. 17, pp 63-79. Amsterdam: Academic.

Blanchard DC, Blanchard RJ, Tom P, Rodgers RJ (1990) Diazepam changes risk assessment in an anxiety/defense test battery. Psychopharmacology 101:511-518.

Blanchard DC, Hynd AL, Minke KA, Minemoto T, Blanchard RJ (2001) Human defensive behaviors to threat scenarios show parallels to fear-and anxiety-related defense patterns of non-human mammals. Neurosci Biobehav Rev 25:761-770.

Blanchard DC, Griebel G, Blanchard RJ (2003) The Mouse Defense Test Battery: pharmacological and behavioral assays for anxiety and panic. Eur J Pharmacology 463:97-116.

Blanchard RJ, Blanchard DC, Hori K (1989) An ethoexperimental approach to the study of defense. In: Ethoexperimental approaches to the study of behavior (Blanchard RJ, Brain PF, Blanchard DC, Parmigiani S, eds), Vol. 48, pp 114-136. Dordecht, The Netherlands: Kluwer Academic.

Blanchard RJ, Blanchard DC, Weiss SM, Meyer S (1990) The effects of ethanol and diazepam on reactions to predatory odors. Pharmacology, biochemistry, and behavior 35:775-780.

Cooper AJ, Perkins AM, Corr PJ (2007) A confirmatory factor analytic study of anxiety, fear, and behavioral inhibition system measures. J Ind Diff 28:179-187.

Corr PJ (2008) The reinforcement sensitivity theory of personality. Cambridge: Cambridge UP

Deakin JFW, Graeff FG (1991) 5HT and mechanisms of defence. J Psychopharmacol 5:305-315.

Farmer A, Mahmood A, Redman K, Harris T, Sadler S, McGuffin P (2003) A sib-pair study of the Temperament and Character Inventory scales in major depression. Arch Gen Psychiatry 60:490-496.

Gould RA, Otto MW, Pollack MH, Yap L (1997) Cognitive behavioral and pharmacological treatment of generalized anxiety disorder: a preliminary meta-analysis. Behav Ther 28:285-305.

Gray JA, McNaughton N (2000) The neuropsychology of anxiety: an enquiry into the functions of the septo-hippocampal system, Ed 2. Oxford: Oxford UP.

Griebel G, Blanchard DC, Agnes RS, Blanchard RJ (1995a) Differential modulation of antipredator defensive behavior in Swiss-Webster mice following acute or chronic administration of imipramine and fluoxetine. Psychopharmacology 120:57-66.

Griebel G, Blanchard DC, Jung A, Blanchard RJ (1995b) A model of antipredator defense in Swiss-Webster mice: effects of benzodiazepine receptor ligands with different intrinsic activities. Behav Pharmacol 6:732-745.

Griebel G, Sanger DJ, Perrault G (1997) Genetic differences in the mouse defense test battery. Aggress Behav 23:19-31.

Griebel G, Perrault G, Sanger DJ (1998) Characterization of the behavioral profile of the non-peptide CRF receptor antagonist CP-154,526 in anxiety models in rodents. Psychopharmacology 138:55-66.

Kuepper Y, Bausch S, Iffland J, Reuter M, Hennig J (2006) S-Citalopram in neuroendocrine challenge-tests: Serotonergic responsivity in healthy 
male and female human participants. Psychoneuroendocrinology 31: $1200-1207$

Kumari V, Corr PJ (1998) Trait anxiety, stress and the menstrual cycle: effects on Raven's standard progressive matrices test. Pers Ind Diff 24:615-623.

Kumari V, ffytche DH, Williams SC, Gray JA (2004) Personality predicts brain responses to cognitive demands. J Neurosci 24:10636-10641.

Litvin Y, Pentkowski NS, Pobbe RL, Blanchard DC, Blanchard RJ (2008) Unconditioned models of fear and anxiety. In: Handbook of anxiety and fear (Blanchard RJ, Blanchard DC, Griebel G, Nutt D, eds), Vol. 17, pp 81-99. Amsterdam: Academic.

Mackinnon A, Jorm AF, Christensen H, Korten AE, Jacomb PA, Rodgers B (1999) A short form of the positive and negative affect schedule: evaluation of factorial validity and invariance across demographic variables in a community sample. Pers Ind Diff 27:405-416.

McNaughton N, Corr PJ (2004) A two-dimensional neuropsychology of defense: fear/anxiety and defensive distance. Neurosci Biobehav Rev 28:285-305.

Mobbs D, Petrovic P, Marchant JL, Hassabis D, Weiskopf N, Seymour B, Dolan RJ, Frith CD (2007) When fear is near: threat imminence elicits prefrontal-periaqueductal gray shifts in humans. Science 317:1079-1083.

Olsson A, Nearing KI, Phelps EA (2007) Learning fears by observing others: the neural systems of social fear transmission. Soc Cogn Aff Neurosci Adv Acc 2:3-11.

Perkins AM, Corr PJ (2006) Reactions to threat and personality: psycho- metric differentiation of intensity and direction dimensions of human defensive behavior. Behaval Brain Res 169:21-28.

Perkins AM, Kemp SE, Corr PJ (2007) Fear and anxiety as separable emotions: An investigation of the revised reinforcement sensitivity theory of personality. Emotion 7:252-261.

Perkins AM, Cooper A, Abdelall M, Smillie LD, Corr PJ (2010) Personality and defensive reactions: fear, trait anxiety and threat magnification. J Personal, in press.

Spielberger CD, Gorsuch RL, Lushene RE, Vagg PR, Jacobs GA (1983) Manual for the state-trait anxiety inventory: STAI (Form Y2). Palo Alto, CA: Consulting Psychologists.

Stemmelin J, Cohen C, Terranova JP, Lopez-Grancha M, Pichat P, Bergis O, Decobert M, Santucci V, Françon D, Alonso R, Stahl SM, Keane P, Avenet P, Scatton B, le Fur G, Griebel G (2008) Stimulation of the b3Adrenoceptor as a novel treatment strategy for anxiety and depressive disorders. Neuropsychopharmacology 33:574-587.

Tabachnick BG, Fidell LS (2007) Using multivariate statistics, Ed 5. Boston: Allyn and Bacon.

Tan S, Kirk RC, Abraham WC, McNaughton N (1990) Psychopharmacology 101:550-554.

Wade AG, Lepola U, Koponen HJ, Pedersen V, Pedersen T (1997) The effect of citalopram in panic disorder. Br J Psychiatr 170:549-553.

Wolpe J, Lang PJ (1977) Manual for the fear survey schedule. San Diego: Educational and Industrial Testing Service. 